\title{
Cosmic shear from STIS pure parallels
}

\section{Data}

\author{
N. Pirzkal ${ }^{1}$, L. Collodel ${ }^{1}$, T. Erben ${ }^{3,4,7}$, R. A. E. Fosbury ${ }^{1}$, W. Freudling ${ }^{1}$, H. Hämmerle ${ }^{2,3}$, B. Jain ${ }^{5}$,
} A. Micol ${ }^{1}$, J.-M. Miralles ${ }^{1,2}$, P. Schneider ${ }^{2,3}$, S. Seitz ${ }^{6}$, and S. D. M. White ${ }^{3}$

1 ST-ECF, Karl-Schwarzschild Str. 2, Garching bei München 85748, Germany

2 Institut für Astrophysik und Extraterrestrische Forschung der Universität Bonn, Auf dem Hügel 71, 53121 Bonn, Germany

3 Max-Planck-Institut für Astrophysik, Karl-Schwarzschild Str. 1, 85741 Garching, Germany

4 Institut d'Astrophysique de Paris, 98bis boulevard Arago, 75014 Paris, France

5 Department of Physics and Astronomy, University of Pennsylvania 209 S. 33rd Street, Philadelphia, PA 19104, USA

6 Universitäts-Sternwarte München, Scheiner Str. 1, 81679 München, Germany

7 Observatoire de Paris, DEMIRM 61, avenue de l'Observatoire, 75014 Paris, France

Received 20 February 2001 / Accepted 12 June 2001

\begin{abstract}
Following the second HST servicing mission in 1997 when the STIS instrument was installed and the capability for parallel observations was enhanced, a substantial archive of non-proprietary parallel data has been accumulating. In this paper, we discuss the use of unfiltered STIS imaging data for a project that requires deep observations along as many independent lines-of-sight as possible. We have developed a technique to determine which datasets in the archive can safely be co-added together and have developed an iterative co-addition technique which enabled us to produce 498 high-quality, deep images. The principal motivation for this work is to measure the Cosmic Shear on small angular scales and a value derived from these data will be presented in a subsequent paper. A valuable by-product of this work is a set of high quality combined fields which can be used for other projects. The data are publicly available at http://www.stecf.org/projects/shear/
\end{abstract}

Key words. cosmology: observations - techniques: image processing - gravitational lensing

\section{Introduction}

In June 1997, parallel observations using the Space Telescope Imaging Spectrograph (STIS) on the Hubble Space Telescope started to be taken in substantial numbers as the STIS Parallel Survey (SPS) was carried out. The result of the SPS was to generate thousands of relatively short exposure images and slitless spectra taken along many different lines-of-sight. The data were nonproprietary and were made available almost immediately.

We are using the imaging data to investigate the distortion of background galaxies by the gravitational field of the large scale matter distribution in the Universe, also known as Cosmic Shear (Mellier 1999; Bartelmann \& Schneider 2001). This effect was recently detected from the ground (Van Waerbeke et al. 2000; Bacon et al. 2000; Kaiser et al. 2000; Maoli et al. 2000; Wittman et al. 2000; Van Waerbeke et al. 2001) and from space (Rhodes et al. 2001). The typical object sizes that have to be measured are on the order of the seeing size of typical ground-

Send offprint requests to: N. Pirzkal

e-mail: npirzkal@eso.org based observations. Such observations are therefore better carried out using the stable, high spatial resolution that is provided by space-based cameras such as those on HST. Due to intrinsic cosmic variance, this project requires many observations of separate, independent fields along different lines-of-sight, each containing several tens of faint, small background galaxies from which high precision measurements of shapes can be made.

This paper, the first in a series, describes the nature of the available STIS data (Sects. 2.1 and 2.2), the selection of the input images from the pool of existing archived images (Sect. 2.4), and the data reduction that has been performed (Sect. 3) to produce 498 deep, coadded fields. We conclude with a description of the extended object content of the reduced data (Sect. 5). A subsequent paper will demonstrate that the properties of the STIS imaging data, the SPS data in particular, make them very appropriate for an investigation of the Cosmic Shear on small scales, and will present Cosmic Shear measurements derived from these data. All of the co-added images discussed in this paper are publicly available at http://www.stecf.org/projects/shear/. 


\section{Data}

\subsection{STIS}

STIS CCD images provide good depth, excellent resolution and adequate sampling of the telescope point spread function (PSF), making them a prime choice for our Cosmic Shear study. The STIS CCD is sensitive to wavelengths ranging from 2500 to $11000 \AA$ and its field of view is $51^{\prime \prime} \times 51^{\prime \prime}$. The STIS CCD pixel size is $0.05^{\prime \prime}$ and is the result of a compromise between properly sampling the STIS CCD PSF ( $F W H M$ of $0.05^{\prime \prime}$ at $500 \mathrm{~nm}$ ) and having a field of view which is as large as possible. The full imaging field of the STIS CCD can only be used in an unfiltered mode but this results in a high sensitivity. In this mode, called the CLEAR filter mode, the attained throughput is significantly higher, and the bandpass broader, than with other HST instruments such as WFPC2. The limiting magnitude in a 1 hour exposure, for a A0V star, at a signal-to-noise ratio of 5 , reached in the STIS CLEAR filter mode, is $V_{\mathrm{AB}}=28$ while WFPC2 combined with the broad F606W filter only reaches a limiting magnitude of $V_{\mathrm{AB}}=27.2($ Gregg \& Minniti 1997). These numbers show how much more efficient the STIS CLEAR filter mode is at capturing photons. The very broad bandpass does, however, bring some disadvantages, the principal ones being the non-standard photometric band and the substantial variation of the diffraction pattern across the band which results in an effective PSF which depends on object colour. As we will see in Sect. 5, in 1 orbit with the CLEAR filter, STIS can image a sufficient number (20-30) of field galaxies for a Cosmic Shear measurement to be attempted. More detailed information about STIS and its performance can be found in the STIS Instrument Handbook (Leitherer et al. 2000), and in Gregg \& Minniti (1997), Kimble et al. (1998), and Gilliland et al. (1999).

\subsection{STIS parallel survey (SPS)}

The HST observing mode called the Parallel Mode became widely available in June 1997 following the installation of solid state recorders on-board HST during the second servicing mission. In Parallel Mode, more than one instrument can be used at a time: the primary instrument carries out its scheduled science observations while other instruments, the parallel instruments, are also allowed to collect data. The pointings of the parallel instruments are dictated by the pointing of the primary instrument and by the location of each instrument in the telescope focal plane. Parallel observations are implemented on a strict basis of non-interference with the primary instrument, imposing strong restrictions on the exposure times and dithering patterns of parallel observations. When parallel observations are not specifically requested by the user of the primary instrument, "Pure Parallel" observations are carried out which do not have a proprietary period and are made public immediately after they have been taken.
Table 1. Content of the SPS proposals listed in Sect. 2.2. Starting with a total of $2149 \mathrm{CR}-$ SPLIT $=2$ images (not counting grism SPS exposures), 1312 images were identified to also be unbinned, and taken in the CLEAR filter. 1303 of these (9 images were rejected because of cosmetic defects) were used to produce 498 deep, long-exposure time, co-added images.

\begin{tabular}{ll}
\hline Type & Number \\
\hline CR_SPLIT datasets & 2149 \\
CR_SPLIT+Unbinned datasets & 1512 \\
CR_SPLIT+Unbinned+CLEAR datasets & 1312 \\
Selected datasets & 1303 \\
Co-added images & 498 \\
\hline
\end{tabular}

The SPS observing program (e.g., Gardner et al. 1998) was created in 1997. Its goal was to make use of the Pure Parallel Mode opportunities of STIS by taking pure parallel direct and grism observations in an automated, scripted manner any time that another instrument was used and STIS remained available. The actual implementation of the SPS has been under the supervision of the Parallel Survey Working Group at STScI.

Some of the SPS data were taken in the CLEAR filter mode, had a relatively short integration time of a few hundred seconds to minimize the impact of cosmic rays (Smette \& Hill 1999), and were taken in what is called CRSPLIT mode. This mode consists of taking at least two successive images in order to later facilitate the removal of cosmic ray impacts from each image. It also ensures that telescope tracking errors (Sect. 2.3 and Appendix) do not accumulate over long periods of time. During the period from June 1997 to October 1998 (date on which images stopped being taken in CR-SPLIT mode), we found the SPS proposals number 7781, 7783, 7908, 7910, 7911, 8062, 8064 , and 8084 to contain datasets that met our requirements. The number of datasets available is summarized in Table 1. A subsequent Cycle 9 GO program (8562; PI Schneider) is continuing the STIS parallel imaging observations in a non-proprietary mode: the analysis of these data is not included here but will be discussed in another paper.

\subsection{Pointing and tracking precision of SPS}

One should distinguish between two separate telescope pointing errors: the first relates to where the telescope is actually pointing at the time when an image was taken. The second is the ability of the telescope system to keep the telescope pointing exactly at the desired position during the course of an image acquisition.

Having identified an appropriate set of images from the SPS, we remained particularly concerned about the amount of image distortion that could have been introduced by the relative instability of the telescope tracking. There are in fact two distinct effects that can result in less than perfect tracking conditions during an SPS exposure. One is the inherent spacecraft jitter while the other one 
is the problem of differential velocity aberration. The effect of the former is seemingly random and usually leads to a symmetrical widening of the effective instrumental PSF. Differential velocity aberration, on the other hand, introduces a systematic drift of the pointing of parallel instruments (See Appendix A).

Random pointing errors are caused by a whole range of processes within the spacecraft, ranging from thermallydriven jumps to minor cosmic dust impacts. While the absolute accuracy of HST's pointing varies from $0.5^{\prime \prime}$ to $2^{\prime \prime}$, relative astrometry between successive images, taken during a single telescope visit and using the same guide stars, is typically good down to between $0.001^{\prime \prime}$ and $0.050^{\prime \prime}$.

The tracking errors caused by the differential velocity aberration depend on the direction of the observed target relative to the direction of the spacecraft velocity vector. While the pointing of the primary instrument takes this effect into account, parallel instruments are affected by a different amount of velocity aberration since they are at a different position in the telescope focal plane. The resulting un-corrected drift in a 400 second STIS exposure can be as large as $0.005^{\prime \prime}$ (0.1 STIS CCD pixel). A more in-depth discussion of the effect of differential velocity aberration is presented in Appendix A of this paper.

While pointing and tracking errors can be expected during the course of an exposure, one can easily check the quality of an HST observation by using information obtained from the on-board Fine Guide Sensors (FGS) system. Under good conditions, HST observations are obtained while the positions of two guide stars are being monitored by the FGS. This information feeds the closed-loop tracking system of the telescope, but it is also recorded a few times a second and incorporated into the Observation Log files which are made available to users. The position of the guide stars as a function of time can be extracted from the Observation Log, allowing one to check how well the telescope tracked during the course of an exposure. Given those FGS guide star measurements, and given knowledge of focal plane geometry and distortions, one can map any point in the telescope's focal plane, such as an aperture, onto RA and Dec as a function of time, during the course of an observation ("jitter ball"). This was done, for each exposure, and how well the telescope was tracking was checked by determining the magnitude and asymmetry of the "jitter ball".

The jitter data can also be used to determine the accurate relative pointing of a set of images, if those were taken in a single telescope visit and using the same guide stars. This provides a good alternative to simply using the available World Coordinate System (WCS) information stored in the image headers since the latter is only a record of where the telescope should have been pointing and does not indicate where the telescope was actually pointing during the observation (Micol et al. 1998). In Fig. 1, we present the result of using the jitter data by showing a histogram of the differences between the WCS coordinates and the coordinates derived using jitter data. We found that $50 \%$ of the WCS-derived offsets dif- fered from the jitter-derived ones by more than 0.3 pixel, while $4 \%$ of them differed by more than 2 pixels.

\subsection{Selecting the SPS datasets}

We restricted our analysis to a sub-set of the available SPS data which satisfies the following conditions: an image had to be taken in the CLEAR filter mode, in a CR-SPLIT mode, be unbinned (with a pixel size of $0.050^{\prime \prime}$ ), and had to have an associated "jitter ball" rms value smaller than $0.005^{\prime \prime}$ (0.1 STIS CCD pixel) (Sect. 2.3). We then determined which images could be combined together to produce deeper images. Starting with all of the jitter data for the time period covered by the proposals we were interested in (Sect. 2.2), we selected images which were taken consecutively during a single telescope visit using the same telescope roll angle, and which were offset by no more than one quarter of the field of view. Such groups of images were logically associated into what we refer to as a STIS Association (Micol et al. 1998) and the relative offsets between the members of each STIS Association were computed using the available jitter data. A histogram of the exposure times of the 1303 individual CR - SPLIT $=$ 2 images that were selected is plotted in Fig. 2.

\section{Reduction procedure}

Since a project such as ours can gain a lot by using deeper images in which more field galaxies can be detected, our data reduction and co-addition procedures were developed to co-add images together without introducing any artificial distortion in the final co-added images.

\subsection{Basic data reduction of individual SPS datasets}

All raw datasets were reduced using the latest version of the standard STIS data reduction pipeline (CALSTIS) and using the best available calibration files. As mentioned in Sect. 2.2, only SPS images taken using a CR - SPLIT = 2 acquisition mode were used for this project, which greatly helped the removal of cosmic ray impacts using just CALSTIS. CALSTIS was also used to bias subtract and flat-field the raw images. The use of the best available calibration files, such as the STIS weekly darks, proved to be a good way to reduce the number of un-corrected STIS hot pixels (pixels with larger than normal dark currents and which affect about $2-3 \%$ of the pixels) by a factor of about two, down to a number of about 1000 .

\subsection{Additional data reduction}

Bad pixel maps, containing the location of un-corrected hot pixels, cosmic rays, as well as the vertical streaks that are associated with those were then created for each of the images processed by CALSTIS.

Hot pixels and residual cosmic ray impacts were located by flagging any pixel which deviated by more than $8 \sigma$ from the surrounding pixels. The group of surrounding 
pixels included all of the adjacent pixels but excluded the pixels in the same column as the pixel being examined since those were often affected by the cosmic ray impact.

Noisy, one-pixel-wide columns (2 to 10 per image) were detected by examining groups of 50 vertically adjacent pixels at a time. When the median of such a column segment was found to be $1 \sigma$ higher than the corresponding column segments in both the preceding and following columns, that particular section, as well as all the pixels below, were flagged as bad. This was shown to accurately detect $95 \%$ of these noisy columns.

No further cleaning or flagging of cosmetic defects in the images, such as spikes and ghosts caused by bright stars (Leitherer et al. 2000) was performed at this stage.

\subsection{Image co-addition}

Once individual images were reduced and their bad pixel maps were generated, the actual process of co-adding the members of a STIS Association was performed using an iterative cross-correlation and Drizzle (Fruchter \& Hook 1998) technique which was specifically developed for this purpose.

The use of Drizzle as the core of this iterative process allowed us to properly exclude bad pixels using the image masks generated earlier (Sect. 3.2), to apply subpixel shifts, to incorporate the known STIS distortion map (Goudfrooij 1997) by un-distorting individual images first, and to project the combined drizzled image onto a finer, sub-sampled grid. A sub-sampling of a factor of two was used in order to reduce the spatial smearing produced when small under-sampled objects are shifted by a fraction of a pixel.

While the offsets derived using the jitter data were more accurate than WCS-derived ones, the use of crosscorrelation allowed us to further refine our estimates of the relative offsets between STIS Association members. The method, which is iterative, proceeds as follows: using the initial offsets determined from the available jitter data, a first estimate of the combined image was generated using the Drizzle IRAF routine. In addition, similarly shifted and drizzled versions of each individual STIS Association member were also produced. SExtractor (Bertin \& Arnouts 1996) was then used to identify objects in both the combined image and the individually drizzled STIS Association members and to produce backgroundfree versions of these images, where background regions were set to a value of zero. The individually drizzled, background-free STIS Association members were then separately cross-correlated with the background-free combined image to determine a set of shift corrections. These were then added to the STIS Association member offsets, and the entire process was repeated using the new offsets estimates until the method converged and the computed shift corrections became smaller than 0.05 pixels. This method usually converged in 4 to 10 iterations.

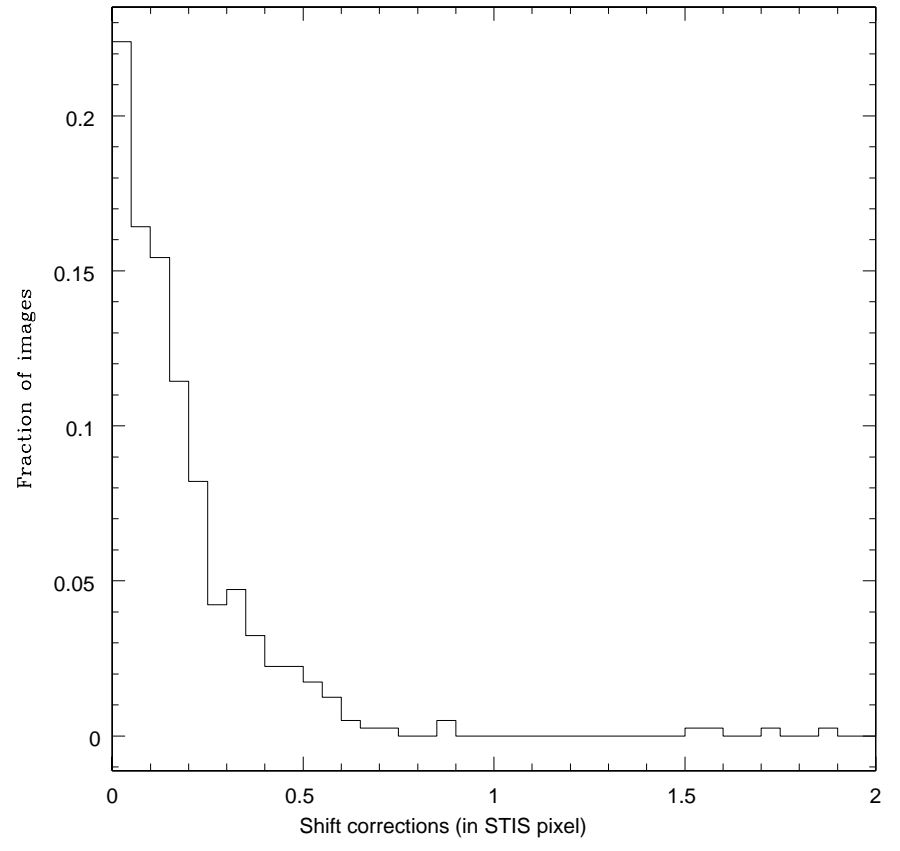

Fig. 1. Histogram of the differences between the values of shifts computed using the available WCS information and the shifts computed using jitter data. This histogram does not include $4 \%$ of the images for which the WCS-derived shifts were off by more than 2 pixels.

\subsection{Resulting output}

Starting with the original pool of datasets from the proposals listed in Sect. 2.2, 1303 individual CR - SPLIT = 2, unbinned, CLEAR filter STIS images were combined together to produce 498 co-added STIS pure parallel Associations.

The effective integration time of the co-added images is a function of the number of members in each STIS Association and of the exposure time of the individual Association members. In Fig. 2, we plot the distribution of exposure times of the individual STIS Association members. The typical offsets between individual members of a STIS Association was 12 STIS CCD pixels. A histogram of the effective exposure times of the combined STIS Associations images is presented in Fig. 3.

\subsection{The applied offsets}

We found that about $4 \%$ of our images had recorded WCS values that were off from the jitter-derived coordinates by more than $0.1^{\prime \prime}$ (2 STIS CCD pixels). Such large offset errors could not have been recovered using our iterative cross-correlation technique which required a reasonably good first estimate of the offsets to start with. Extensive checking of the accuracy of these final images offsets was done and is summarized in the next Sect. 4. 


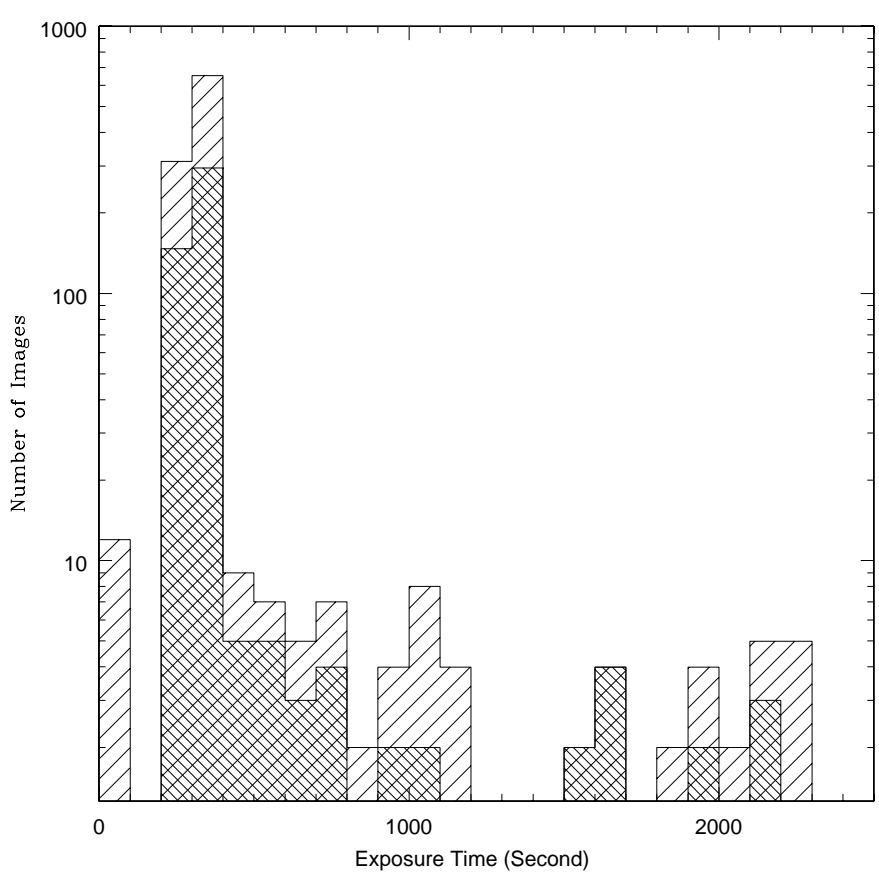

Fig. 2. Histogram of the exposure times of the individual STIS Pure Parallel images (coarse hashes). Each image is made up of two separate exposures, each with half of the exposure time $(\mathrm{CD}-\mathrm{SPLIT}=2$ mode $)$. The subset of images with galactic latitude greater than 30 degrees is also plotted (tight hashes).

\section{Testing of the co-addition procedure}

The reduction and image co-addition procedure outlined in Sect. 3.3 was thoroughly tested by applying the technique to a set of simulated STIS Associations.

We created simulated STIS Associations containing objects with positions, magnitudes, shapes, and spatial distributions realistically modeled after the content of one of our high galactic latitude SPS field containing 75 objects. We used Tiny Tim ${ }^{1}$ to generate the STIS PSF which we used to produce our simulated images.

As a test, 20 simulated STIS Associations, each containing 6 dithered member images, were constructed. In each of these simulated Associations, we varied the actual offsets between each Association member, assigning them realistic values. Poisson noise, background sky, and a realistic number of cosmic ray impacts were added using the IRAF tasks MKOBJECTS and MKNOISE. These simulations were processed using the procedure outlined in Sects. 3.2 though 3.3. In every single one of our 20 simulations we were able to re-align the individual STIS Association members to within $0.001^{\prime \prime}$ (0.02 STIS CCD pixel).

This test was then repeated a second time using a new set of 20 simulated STIS Associations. This time however the 14 brightest objects amongst the 75 objects used initially were removed. We also generated Associations with different $S / N$ by arbitrarily increasing the magnitudes of the 61 objects in our simulated fields, up to

${ }^{1}$ http://www.stsci.edu/software/tinytim/tinytim.html

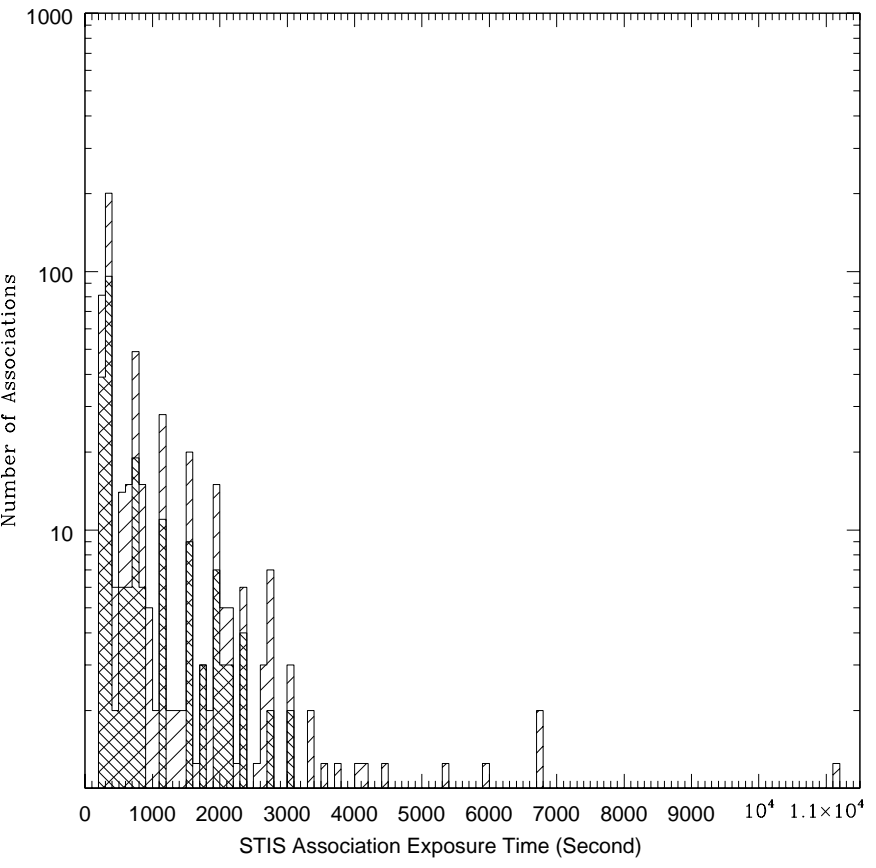

Fig. 3. Our STIS Associations effective exposure times (coarse hashes). Associations with galactic latitude larger than 30 degrees are also plotted (tight hashes).

0.7 mag per object. Some Associations were also generated with and some without cosmic rays or hot pixels to verify that our cosmic rays and hot pixel removal method (see Sect. 3.2) did not influence our offset determinations. Our co-addition method proved very efficient, and the individual STIS Association member offsets were re-constructed. Even in the case of simulations with objects having a signal to noise ratio which was half of the value measured on the observed image, we were able to recover the proper offsets to within $0.004^{\prime \prime}$ (0.08 STIS CCD pixel).

We additionally tested the sensitivity of our method to the object-blurring effect that the STIS Charge Transfer Inefficiency (CTI) might cause as the centroid of objects located at low $Y$ coordinates is progressively shifted to lower $Y$ coordinates relative to object higher up in the CCD. We tested this by selecting one of our STIS associations containing a large number of members $(14$ members of $300 \mathrm{~s}$ each, with relative offsets of as much as 40 pixels), and determined the shifts separately for the top and bottom halves of the images. This test was made once using only the brighter (10 per half field), and once more using only the fainter objects (5 connected STIS CCD pixels $3 \sigma$ above the background). In all cases, the derived offsets were consistent (to within $1 / 20$ of a STIS CCD pixel) with the offsets derived using all the objects in the entire image. This shows that the STIS CTI does not affect our ability to properly determine the offsets between STIS association members. The test using only bright objects additionally shows that our image cross correlation is not significantly affected by the presence of STIS PSF ghosts, which previously described tests using Tiny Tim could not include. 


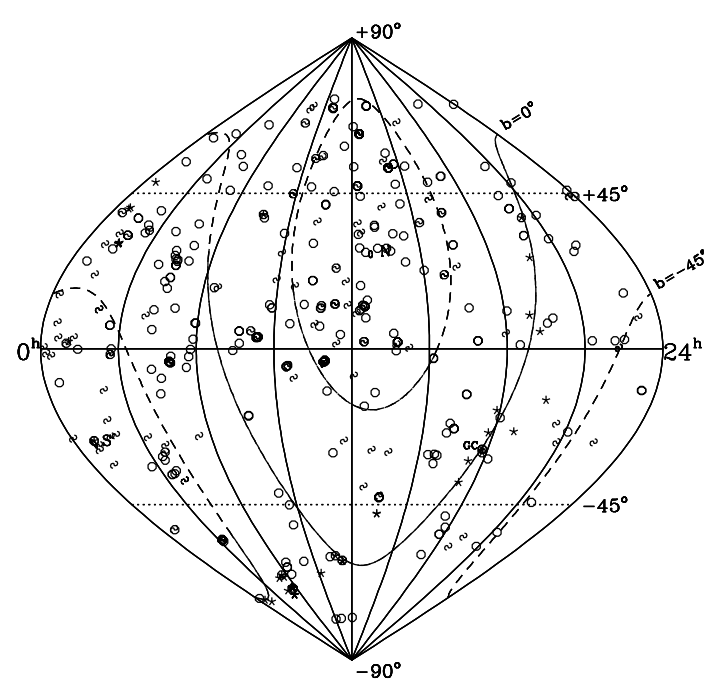

Fig. 4. Galactic coordinates of the 498 co-added fields. The stars, swirls, and empty circles represent stellar fields, galaxy fields, and non-classified fields respectively (See Sect. 5.2).

While flux conservation was also tested, we do not present the test results in this paper because they only confirm the known ability of the Drizzle algorithm to conserve flux (Fruchter \& Hook 1998). We confirmed through these tests that our method preserves the shape of individual objects. An extensive description of these results will be presented in the second paper of this series.

\section{Sky coverage, number counts, and galaxy sizes}

\subsection{Sky distribution, exposure times and magnitude limits}

The 498 fields that we produced from the STIS SPS data are spread around the sky. Figure 4 shows the spatial distribution of our datasets.

Our 498 co-added images have a very heterogeneous distribution of exposure times, and the limiting magnitude reached on a field depends on the number of images present in the STIS Association and on the integration time of the individual images. The final distribution of exposure times is plotted in Fig. 3. Using SExtractor, we estimate our limiting magnitude, for a 5 pixel detection and for a signal to noise of 3.0, to be $M_{\mathrm{AB}}(\mathrm{CLEAR})=28.5$ in a 1 hour exposure.

\subsection{Galaxy number counts}

Our final co-added images are distributed all over the sky and are likely to be affected by various amounts of galactic absorption. While this implies our sample of images is not adequate for a detailed study of galaxy number counts, number counts provide an interesting description of our data.

All of the co-added Associations were visually inspected and classified as being possibly a member of two possible types of fields: galaxy or stellar fields. Galaxy

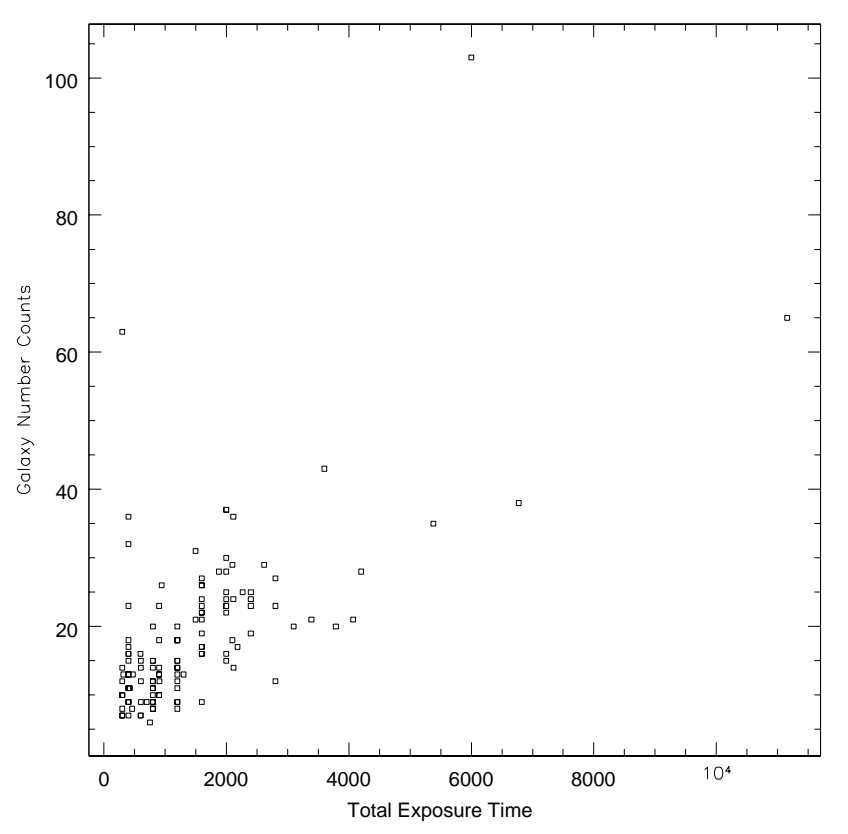

Fig. 5. Number of detected galaxies per STIS SPS Association plotted as a function of exposure time, for the 122 galaxy fields.

fields are fields where more than 10 extended objects were detected with the procedure described below while stellar fields are fields where more than 100 point like objects, uniformly distributed over the field, could be detected. Starting with the 498 co-added STIS Associations, 55 stellar fields and 122 galaxy fields were identified. SExtractor was used to generate an object catalog of the galaxy fields. The main detection criteria used during the extraction process were that each object had to have a minimum of 5 pixels at least $3 \sigma$ brighter than the local background. A Gauss_4.0_7 $\times 7$ convolution kernel was used. The complete SExtractor parameter file is being made available at http://www.stecf .org/projects/shear/. As seen in Fig. 5, the number of detected galaxies per co-added image rises steadily from $12 \pm 6$ to $29 \pm 12$ for total exposure times ranging from 500 to $2500 \mathrm{~s}$. The galaxy number counts then rises more slowly as the exposure time increases past $2500 \mathrm{~s}$. This flattening trend is caused by the intrinsic flattening of the galaxy number counts at increased magnitude and because distant (and/or faint) objects quickly become too small to be resolved by STIS (see Figs. 6 and 1 in Gardner \& Satyapal 2000) and to be classified as galaxies. This leads to the conclusion that the optimal integration time for a project using STIS which requires as many field galaxies in as many independent lines of sight as possible is somewhere between 2000 to $2500 \mathrm{~s}$, which corresponds to approximately 29 galaxies per field.

It should be noted that the redshift distribution of these objects, which will be needed for the quantitative interpretation of our Cosmic Shear measurement, can not be derived using solely the STIS SPS data. This information will however be determined using photometric redshifts 


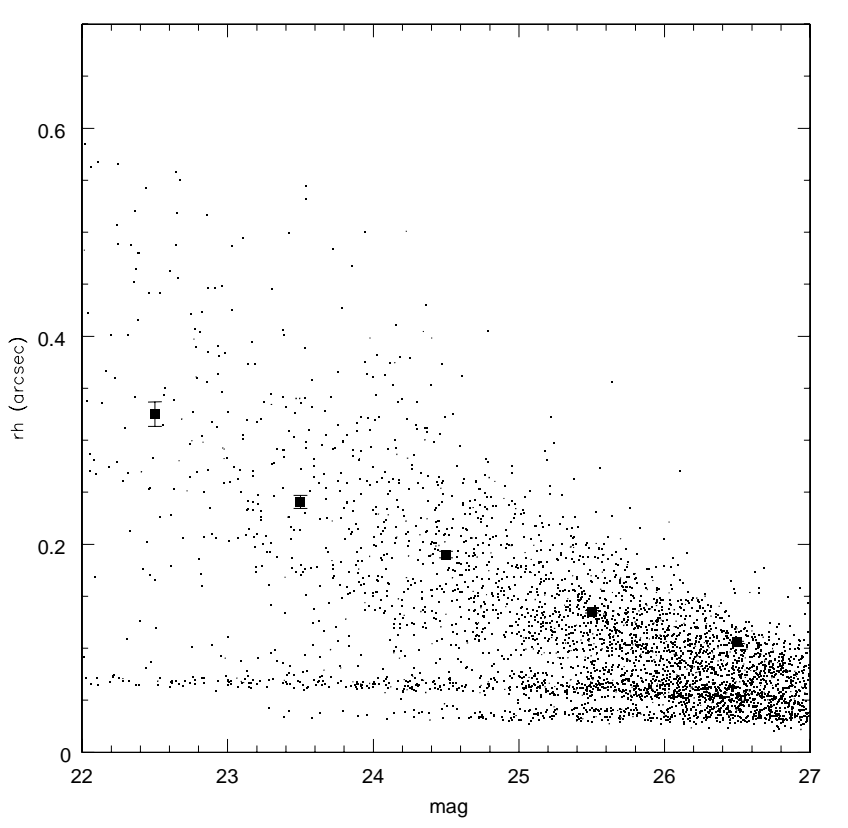

Fig. 6. Measured half-light radius of objects in our co-added STIS SPS images. The horizontal distribution of points at a half-light radius between $0.05^{\prime \prime}$ and $0.08^{\prime \prime}$ are caused by stars and other un-resolved objects. The lowermost horizontal distribution of points with a half-light radius ranging from $0.025^{\prime \prime}$ to $0.030^{\prime \prime}$ is caused by spurious detections of single noisy pixels by SExtractor. The average sizes of galaxies per magnitude bin are also indicated. The filled squares mark the average sizes of objects at each magnitude, counting only those with half-light radius $>0.08^{\prime \prime}$. The error bars represent the $1 \sigma$ level in the error of the mean.

obtained from lower resolution, deep, multicolor VLT images.

\subsection{Galaxy sizes}

While our co-added STIS SPS Associations provide deep, high resolution images, it can be seen in Fig. 6 that the average size of galaxies decreases as object CLEAR filter $\mathrm{AB}$ magnitudes increase. We estimate that objects with magnitudes ranging from 25.5 to 26.5 have average sizes ranging from $0.14^{\prime \prime}$ to $0.11^{\prime \prime}$. This result is consistent with the results published by Gardner \& Satyapal (2000) which was based on 115 STIS observations of the HDF-S, and with results from Odewahn et al. (1996). From our SPS data, one can expect most galaxies with magnitude ranging from 22 to 26 to have half-light radii ranging from $0.3^{\prime \prime}$ to $0.1^{\prime \prime}$ (6 to 2 STIS CCD pixels). The upper right portion of Fig. 6 is however expected to suffer from incompleteness since it is likely to be affected by our relative insensitivity to low surface brightness objects.

\section{Conclusion}

We have begun an investigation of Cosmic Shear which requires many images at different pointings containing a high number of faint field galaxies. One source of such data has been provided by the SPS program which has been producing a considerable amount of data since June 1997. We have successfully accumulated the SPS data taken from June 1997 to October 1998 and have used HST jitter data to co-add as many of these images as possible. In this way, we have produced a set of 498 co-added images which have individual integration times ranging from 400 to $11000 \mathrm{~s}$. The images were co-added using an iterative cross correlation technique that we have developed and which was shown using simulations to be able to consistently match images to within 0.05 STIS CCD pixel.

We selected a set of 122 images which contains a sufficient number (10 to 30 ) of extended objects to make a meaningful Cosmic Shear measurement. The number counts of galaxies in these images are consistent with previously published numbers (Gardner \& Satyapal 2000), and the optimal STIS integration time is between 2000 and $2500 \mathrm{~s}$, leading to an average of 29 galaxies to be detected at the $3 \sigma$ level. The average sizes of the galaxies with magnitude ranging from 22 to 26 vary from $0.3^{\prime \prime}$ to $0.1^{\prime \prime}$ and are also consistent with previous observations (Gardner \& Satyapal 2000; Odewahn et al. 1996).

The STIS galaxies have presumably a wide redshift distribution, which is essentially unknown at this point. In order to transform a Cosmic Shear measurement on the STIS images into a cosmological constraint, we are currently carrying out lower resolution, deep VLT images to determine the photometric redshift distribution of the STIS galaxies.

The analysis of these fields will be presented in Paper II. The co-added data described in this paper is made available at

http://www.stecf .org/projects/shear/

Acknowledgements. We thank the staff of the STScI for their hard work which, as a response to suggestions made by the Parallel Working Group in 1997, enabled these observations to be carried out. We also thank the two referees for their helpful comments. This work has been partly supported at the ST-ECF by the ESO Director's Discretionary Fund, the TMR Network "Gravitational lensing: new constraints on cosmology and the distribution of dark matter" of the European Community, and by a grant from the Verbundforschung Astronomie/Astrophysik.

\section{Appendix A: Differential velocity aberration}

In this appendix, we investigate the effects of differential velocity aberration on a parallel observation.

The motion of an observer relative to an observed object causes an apparent displacement of the target on the sky with respect to its real geometrical position due to velocity aberration. Since the velocity vector changes during the course of an observation, corrections must be continuously applied to the telescope tracking system. These corrections must be computed for a given line-of-sight, such as the center of the field-of-view of a given instrument in the case of HST. All the other lines-of-sights will be left partially uncorrected, leading to what is referred to as differential velocity aberration. This effect is much larger 

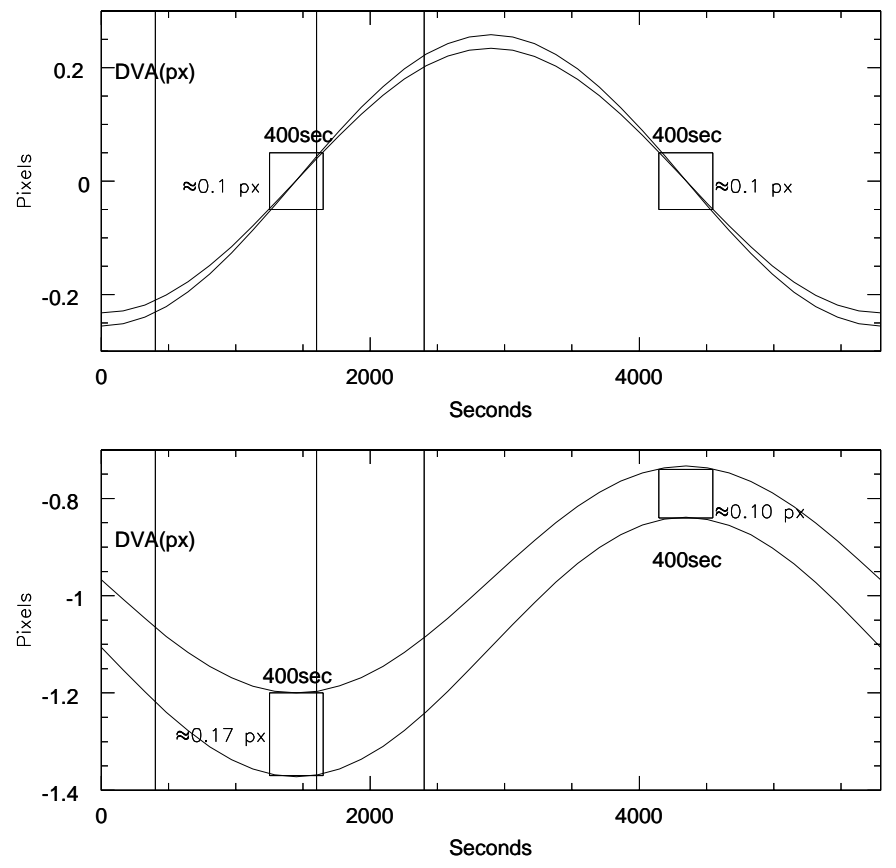

Fig. A.1. The differential velocity aberration (DVA; in STIS CCD pixel) as a function of HST orbital period (97 min), in two extreme situations (see text). The two lines show the result for two points laying on opposite STIS corners. The vertical lines mark some typical STIS Parallel exposure times. The boxes represent individual $400 \mathrm{~s}$ exposures.

for HST than for a ground-based observation since HST travels at a larger speed.

A first effect of differential velocity aberration is an overall tracking error of the secondary, parallel instrument, which leads to an elongated image. A second effect is caused by the difference in velocity aberration from one corner of the field-of-view of the instrument to the opposite corner, leading to what appears to be small change in the image scale over the field of view.

The effect of differential velocity aberration on SPS data can be investigated by studying what happens in the worst possible case: when STIS is being used in Parallel Mode while NICMOS is the primary instrument. This is the worst case since the separation between these two instruments in the HST focal plane is greater than the one between STIS and any other instruments on-board HST. Two extreme cases can then be examined: HST moving in the same direction as the line-of-sight, and HST moving perpendicularly to the line-of-sight. We assume that HST's orbit lies in the ecliptic plane and that both the geocentric velocity of the spacecraft and the velocity of the Earth around the Sun are constant $\left(7\right.$ and $29 \mathrm{~km} \mathrm{~s}^{-1}$ respectively).

The upper panel in Fig. A.1 shows the case of a line-ofsight that is perpendicular to the direction of the telescope motion. The lower panel shows the case of the line-ofsight that is exactly aligned with the velocity vector of the telescope. We have overlaid three vertical lines to mark time intervals of 400, 1600 and $2400 \mathrm{~s}$. These represent typical total exposure times of individual SPS images and of our STIS Associations containing 4 and 6 consecutive SPS observations.

On one hand, the amount of shift that can be expected between a set of successive $400 \mathrm{~s}$ exposures is given by the amplitude of either of the two curves in each panel. During the course of a full orbit, the resulting relative shifts between consecutive images can be as much as 0.5 STIS CCD pixel $\left(0.025^{\prime \prime}\right)$, if no extra dithering of the primary instrument is done. On the other hand, the two curves in each of the panel of Fig. A.1 show the differential velocity aberration for two targets located on opposite corners of the STIS detector. The difference between these two curves therefore provides an estimate of the image scale variation in a STIS frame, which can be seen to vary from 0.1 to 0.17 STIS CCD pixel in the case of $400 \mathrm{~s}$ exposures. This effect is quite small, but its effect on the co-addition of images that are offset with respect to one another can be reduced by restricting the amount of shifts allowed between images to be co-added, as we have done for this project by limiting the maximum allowed shifts to be $1 / 4$ of the field-of-view (Sect. 2.4).

\section{References}

Bacon, D., Réfrégier, A., \& Ellis, R. S. 2000, MNRAS, 318, 625 Bartelmann, M., \& Schneider, P. 2001, Phys. Rep., 340, 291

Bertin, E., \& Arnouts, S. 1996, A\&AS, 117, 393

Fruchter, A. S., \& Hook, R. N. 1998, submitted to PASP [astro-ph/9808087]

Gardner, P., Hill, R. S., Baum, S. A., et al. 1998, ApJ, 492, L99

Gardner, P., \& Satyapal, S. 2000, AJ, 119, 2589

Gilliland, R. L., Goudfrooij, P., \& Kimble, R. A. 1999, PASP, 111, 1009

Goudfrooij, P., Beck, T., Kimble, R., \& Christensen, J. A. 1997, STIS Instrument Report, 1997-10

Gregg, M. D., \& Minniti, D. 1997, PASP, 109, 1062

Kaiser, N., Wilson, G., \& Luppino, G. 2000, submitted to ApJL [astro-ph/0003338]

Kimble, R. A., Woodgate, B. E., Bowers, C. W., et al. 1998, SPIE, 3356, 188

Leitherer, C., et al. 2000, STIS Instrument Handbook, Version 4.1, Baltimore, STScI, [http://www.stsci.edu/instruments/stis]

Maoli, R., Van Waerbeke, L., Mellier, Y., et al. 2001, A\&A, 368,766

Mellier, Y. 1999, ARA\&A, 37, 127

Micol, A., Pirenne, B., \& Bristow, P. 1998, Astronomical Data Analysis Software and Systems VII, ed. R. Albrecht, R. N. Hook, \& H. A. Bushouse, ASP Conf. Ser., 145, 45

Odewahn, S. C., Windhorst, R. A., Driver, S. P., \& Keep, W. C. 1996, ApJ, 472, L13

Rhodes, J., Refregier, A., \& Groth, E. J. 2001, ApJ, 552, L85

Smette, A., \& Hill, R. S. 1999, GSFC STIS Analysis Report Number 60

Van Waerbeke, L., Mellier, Y., Erben, T., et al. 2000, A\&A, 358,30

Van Waerbeke, L., Mellier, Y., Radovich, M., et al. 2001, A\&A, submitted [astro-ph/0101511]

Wittman, D. M., Tyson, J. A., Kirkman, D., Dell'Antonio, I., \& Bernstein, G. 2000, Nature, 405, 143 